\title{
Reflections on My First Year as Oral Pathology Section Editor: "The Good, the Less Than Ideal and the Downright Ugly"
}

\author{
Paul C. Edwards \\ 2012 \\ Oral Surgery, Oral Medicine, Oral Pathology, Oral Radiology, 114(2), 143-146
}

As I pass the one-year milestone in my new role as oral and maxillofacial pathology section editor, I thought that this would be an opportune time to step back for a moment and reflect on my experiences to date. Taking artistic license with the title of the epic 1960s movie, directed by Sergio Leone, lets take a closer look at the highlights:

\section{"The Good":}

The most obvious observation that I have made over the past year is that the success of the oral pathology section of this journal (I am confident that the other section editors would agree that this is true of their sections as well), is indisputably dependent on the hard work and contributions of a very large number of individuals, many of who "work behind the scenes". I list these individuals in no particular order, and apologize in advance if I inadvertently leave anyone out.

First and foremost, recognition must go to the large number of authors from all over the world who continue to submit high quality manuscripts to the oral pathology section, even with the reasonable probability of receiving an unfavorable decision. Due to the high volume of submissions received, only $10 \%$ of submissions are ultimately accepted for publication, although this varies by manuscript type (with original studies and case series having a much higher acceptance rate than case reports; more on that below).

I would like to take this opportunity to let potential authors, and authors who may have submitted manuscripts that ultimately were not accepted, know that a decision not to accept your manuscript for publication should not be considered a negative reflection on the quality of your research or manuscript. In many cases, high quality studies are rejected for publication because they are not of direct relevance to the core readership of this section, the majority of whom are oral and maxillofacial pathologists or individuals with a very strong interest in this field

The same applies for single case reports. Many journals have made the decision to no longer accept case reports. This approach has its basis in many factors, not the least of which is the increasing volume of high quality studies and case series received for publication and the limited space and high production costs associated with the publication of print journals. In addition, the high workload involved in screening, and where appropriate, reviewing large numbers of case reports, especially in light of the potential effect of large numbers of case reports on a journal's overall impact factor, while not primary deciding factors, must also be taken into consideration. On a personal level, I believe that a well-written and timely case report can make a very valuable contribution to the oral pathology literature. As a young resident, I relied heavily on case reports to guide the development of my knowledge base in the field of oral and maxillofacial pathology. Even today, I will routinely reference a case report or two when faced with unusual clinical or 
histopathologic situations. Fortunately, 0000 still accepts case reports for online publication. These are listed in the table of contents of the print version of the journal, and are fully indexed via PubMed. However, only case reports of exceedingly unusual conditions or those with atypical presentations are typically considered for publication.

Authors can increase their likelihood of a favorable decision by targeting their manuscript for submission to the appropriate journal. As stated in the Section Scope Statements of the Guide To Authors ${ }^{1}$, and worth repeating here: "the oral pathology section encourages the submission of original articles of high scientific quality that investigate the pathogenesis, diagnosis, and management of diseases affecting the oral and maxillofacial region. Submitted manuscripts may summarize findings from clinical, translational or basic research in the broad field of oral and maxillofacial pathology, but must contribute substantively to the body of knowledge in this field and should be of obvious clinical and/or diagnostic significance to the practicing oral and maxillofacial pathologist. Areas of focus may include the investigation of disease pathogenesis, the diagnosis of disease using microscopic, clinical, radiographic, biochemical, molecular or other methods; as well as the natural history and management of patients with various conditions of the head, neck and oral mucosal structures. Articles presenting novel and reproducible research that introduce new knowledge and observations are especially encouraged. This section also welcomes the submission of topical review papers on relevant subjects."

Confirmatory articles that largely reproduce previously published studies, while important to the scientific literature (the Sudbho situation ${ }^{2}$ immediately comes to mind), are sometimes more challenging to get accepted for publication. To increase the likelihood of obtaining a favorable decision, these manuscripts should ideally represent either noticeably larger studies, studies that use substantially different methodologies to investigate the same question, or studies that call into dispute the accepted dogma.

Case series, especially when these are large, often multi-centered by necessity, studies directly related to oral pathology, are generally favorably received, particularly if they offer insight into a novel aspect of a condition or process. It goes without saying that well-planned original investigations remain the mainstay of journals such as 0000.

The many reviewers who generously volunteer to spend significant time reviewing submitted manuscripts are the backbone of specialized peer reviewed journals such as 0000. I would like to take this opportunity to thank all of the individuals who have, over the past year, contributed to the success of our Academy's journal by graciously offering their time and expertise to review submissions. While the review comments may on occasional appear somewhat exacting to the authors who have put in a great deal of effort to construct the study and ultimately compose the manuscript, it has been my observation that our reviewers are an extremely dedicated group of professionals who take their responsibilities very seriously; 
namely upholding the high quality of the oral pathology section of 0000 and offering constructive feedback, that, if accepted by the authors, will generally enhance the quality and readability of the manuscript in question. I, for one, have, on many occasions, been thankful for reviewer suggestions of manuscripts that I have submitted for publication. These recommendations, while often requiring significant revision and additional experimental data, have invariably made for a stronger manuscript in the end.

Editorial board members are called upon to review a large number of manuscripts, typically 3 or more per month, as well as to provide guidance to the section editor, and should be recognized and thanked for their contributions. Additionally, Kurt Summersgill needs to be recognized for the terrific job he has been doing managing the clinical pathologic conference section.

Under the outstanding direction of Mark Lingen, the support of our colleagues at Elsevier (Jane Ryley, publisher; Alice Landwehr, managing editor; Jill Shepherd, journal manager) as well as the other section editors (Craig Miller, David Precious, Bill Scarfe), and all of the individuals previously mentioned, it is clearly apparent that the priority of all involved is to further enhance the already strong reputation of this journal.

The decades long affiliation between the oral pathology section of this journal and the American Academy of Oral and Maxillofacial Pathology (AAOMP) is irreplaceable. As the official journal of the AAOMP, the support of the Academy and its members has been a critical aspect of the growth of this section of the journal. Likewise, Janet Svasas, executive director, and Karen Benton, administrative director, at the AAOMP head office, deserve recognition for their assistance.

\section{"The Less Than Ideal"}

While "the good" far outweighs "the less than ideal", or "the downright ugly", there have been several interesting dilemmas over the past year.

Although fortunately a rare occurrence, it can be a challenge reconciling widely differing opinions on the validity and suitability of a particular manuscript for publication. This brings into conflict the two roles that I view as my principal duties as section editor: to act as an advocate for the author, who has spent a great deal of effort in designing and performing the study and preparing it for publication, while ensuring that the high standards of this section are maintained. In those cases, I will typically rely on additional input from members of the editorial board.

Although I can offer no concrete evidence to support this contention, anecdotally at least, it would appear that the ease with which manuscripts can now be submitted electronically has resulted in an increase in the number of papers submitted prior to being thoroughly proof read. These vary from half page case reports with no discussion, to meandering manuscripts that lack critical assessment of the study's 
findings. Sometimes, I can't help but wonder if the occasional author is hoping that the reviewers and section editor will take on this role.

I am grateful to our reviewers, who have identified a number of instances in which publications that would have been more appropriately written as single publications were instead subdivided into several manuscripts for submission to multiple journals (e.g. an article focusing on a particular method employed was submitted to 0000, while the actual findings from the study, presumably along with another overview of the methods, were submitted to a separate journal). Referred to colloquially as "salami slicing" 3 , this is a questionable practice at best, and at worst a strain on the editorial review process.

An occasional challenge involves how to address the single case report or smaller study that lists an extensive number of authors. The desire to recognize all of those involved in the management of a particular case or study is certainly understandable. However, if I could offer any advice to prospective authors, it would be to carefully define the role of each author in the planning and implementation of the study, as well as in the final preparation of the manuscript. If each of the authors played a meaningful and definable role (not just involving the management of the patient, as in the single case report), then 7-10+ authors could conceivably be perfectly justifiable.

\section{"The Downright Ugly"}

It should come as no surprise that plagiarism and fraud constitute the principal breach of trust under the category of "downright ugly". A quick count identified at least 25 submissions that were rejected in the oral pathology section of this journal because of plagiarism within the last 12-month period alone. This figure may underrepresent the number of actual cases, as it only includes manuscripts that were considered or ultimately sent out for peer review, and does not include the approximately $70 \%$ of all submissions that are editorially rejected. Fortunately, in the big picture, these instances of plagiarism still represent only a small fraction of the total number of manuscripts submitted. But obviously even a single case is too many.

How were these cases identified? Most were recognized by sharp-eyed reviewers, who in many cases were suspicious of differences in sentence structure between paragraphs and confirmed the presence of plagiarized text, often with the assistance of internet search engines (e.g. a Google search of the suspected sentence). In at least one case, a reviewer recognized the plagiarized text as his own.

Other cases are identified by "plain old good luck". Two instances come to mind. While reviewing a recent submission, I recognized several familiar passages from a manuscript that I had previously accepted, and that had just been published two months earlier in 0000. On further review, it turned out that large parts of this newly submitted manuscript had been plagiarized from other published sources. This approach, in which the entirety of the introduction and discussion sections 
consists of 1-4 sentences that have been copied verbatim from other sources, is a recurring theme among these cases. What is even more disconcerting is the not uncommon observation that an internet search of the text in question identifies the exact same sentences in more than one previously published manuscript.

The second case was even more fortuitously discovered. While performing a brief literature search after coming across a biopsy of a somewhat uncommon lesion that had just been received from a contributor, I downloaded a 2-year-old reference from another journal. On reading this previously published case report, it was immediately evident that large parts of the text were eerily similar to a recently accepted manuscript for which I had just reviewed the final proofs (one of the last steps prior to final publication). On further review, the entire introduction, discussion, and even large parts of the case presentation section, including the radiographic description, which on initial submission had curiously not matched the particulars of the actual case submitted (and in retrospect should have tweaked my suspicion), had been almost entirely plagiarized from this two-year old article. In this situation, it was apparent that a single coauthor, who had been responsible for writing the plagiarized sections, was solely responsible. If nothing else, this should be taken as a cautionary note to potential coauthors to carefully scrutinize, both for possible plagiarism and for the validity of the underlying scientific data, all manuscripts that you are contemplating placing your name on.

Several other instances come to mind. While serving as section editor of the Clinical Pathologic Conference (CPC) section of this journal several years ago, we received a letter from a concerned individual advising us that a CPC manuscript we had just printed had been previously published as a case report in a non-PubMed referenced print journal ${ }^{4}$. After confirming the allegation of a "duplicate publication", the article was retracted 5 .

Within the past year, two examples of "redundant papers", in which submissions that were very similar to papers previously published by the same authors, the principal differences being the inclusion of minor amounts of additional data or a slightly different analysis of the previous data, were identified during the review phase. Interestingly, both of these submissions also contained images identical to those in the previously published manuscripts.

Some cases are not as clear-cut. In on instance, on closer examination of two photos purporting to show the preoperative and 3 year post-operative clinical presentation of a patient with a cheek mass, it was noted that the patient was wearing the same sweater in both images, along with an identical haircut. The only difference between the two images was the presence of a 2-3 day additional growth of facial hair in the follow-up photograph. Was the second image taken 3 year later? That was certainly possible. Or is it conceivable that it was taken 3-4 days later, at the first postoperative follow-up? Fortunately, in this case, the reviewers legitimately disagreed with the histopathologic diagnosis, and the manuscript was denied. 
These examples are in no way a phenomenon unique to our journal or discipline. As an ad hoc reviewer for numerous other journals, I come across manuscripts with extensively plagiarized sections of text on a routine basis.

Many justifications have been put forth to explain these lapses in judgment. A common premise is that these ethical violations are a direct consequence of the pressure to "publish or perish". While I think there is some validity to this, especially as it relates to data manipulation, it does not adequately account for those authors who "cut and paste" large sections of text verbatim from other sources. Does it really take that much longer to review a body of knowledge and then summarize it in one's own words? An alternative argument that is sometimes offered is this could be related to the authors' limited comfort with writing scientific manuscripts in the English language. Again, there are several resources listed for authors ${ }^{6}$, both publisher affiliated and non-publisher affiliated that offer both English language editing and translation services at, in my opinion, extremely reasonable rates. Moreover, some of the most egregious examples of plagiarized text have come from authors whose native language is English.

Occasionally, the contrarian viewpoint is raised that the use of text copied verbatim from another source is not necessarily of major significance and can even be considered a forme fruste of recognizing the high regard to which the offending author views the work of the original authors (that assumes that the authors have referenced the source of the copied text, which is often not the case). This begs the obvious question: if the authors "took the easy way out" by cutting and pasting large sections of text from other articles, how much can the rest of their data be fully trusted? It also brings into question how the potential author would not anticipate, even succeeding in getting their manuscript published, ultimately being exposed, particularly in this digitally interconnected society and in such a highly specialized field of study.

Under the leadership of Mark Lingen, the editor of this journal, and Jane Ryley, the publisher, plagiarism software is now available to all section editors. This will reduce the number of manuscripts with plagiarized text making it to publication, but will not have a direct effect on instances of scientific "laxity". That is of course where the peer review process comes into play. Ultimately, though, the role of the section editors cannot be that of journal Sheriff. In the end, although we must always be on the lookout for the possibility of ethical lapses, we must still primarily rely on the character and integrity of the vast majority of scientists and clinicians who submit their manuscripts for publication. As frequently quipped by former U.S. President Ronald Regan, "Trust, but verify".

\section{Final Thoughts}

As a final comment, I would encourage potential authors with novel studies or interesting case series to give strong consideration to sending your manuscripts to 0000. A journal's reputation is critical when considering whether to submit an article to a particular journal. With that in mind, it is worth reiterating, as described 
by Craig Miller ${ }^{7}$ in a recent editorial in this journal, and further explored in a study in the Journal of Dental Research ${ }^{8}$, that certain metrics may offer a more accurate assessment of a journal's reputation when comparing highly focused medical-dental specialty journals. Looking at the Eigenfactor Score, for example, which assigns weight scores to the source of the citation as opposed to simply the total number of citations, 0000 is ranked $4^{\text {th }}$ place among all dental journals (Dec. 31, 2009).

Moreover, considering the many countries from which our manuscript submissions originated within the past year alone (Australia, Brazil, Canada, Chile, China, Columbia, Croatia, Czech Republic, Egypt, France, Germany, Greece, Hungary, India, Iran, Israel, Italy, Japan, , Libya, Mexico, New Zealand, Nigeria, Pakistan, Poland, Portugal, Qatar, Saudi Arabia, Serbia, South Africa, South Korea, Spain, Sri Lanka, Taiwan, Thailand, Tunisia, Turkey, United Kingdom, United States, Venezuela within the last year alone), it is readily apparent that this truly is a journal with wideranging international recognition among professionals in the fields of oral surgery, oral pathology, oral medicine and oral radiology.

Paul C. Edwards

Section editor, oral and maxillofacial pathology 


\section{References:}

${ }^{1}$ http://www.elsevier.com/wps/find/journaldescription.cws_home/727585/author instructions. Accessed April 30, 2012.

${ }^{2}$ Gerber P. What can we learn from the Hwang and Sudbo affairs? MJA 2006;184:632-5.

${ }^{3}$ The cost of salami slicing. Nature Mater 2005; 4:1 (Editorial)

${ }^{4}$ Ramani P, Chandrasekar T, Anuja N, Muthusekar MR, Sherlin HJ, Kulkarni A. A swelling in the buccal mucosa with intracranial involvement._Oral Surg Oral Med Oral Pathol Oral Radiol Endod 2007;103:308-13.

${ }^{5}$ Retraction notice. Oral Surg Oral Med Oral Pathol Oral Radiol Endod 2008;10:463. ${ }^{6}$ http://webshop.elsevier.com/languageservices/index.cfm. Accessed April 30, 2012.

7 Miller C. Impact versus impact factor and Eigenfactor. Oral Surg Oral Med Oral Pathol Oral Rad 2012;113:145-6.

${ }^{8}$ Sillet A, Katsahian S, Rangé H, Czernichow S, Bouchard P. The Eigenfactor score in highly specific medical fields: the dental model. J Dent Res 2012;91:329-333. 